\title{
Characteristics and Transmission Dynamics of COVID-19 in Resident Physicians at a Mexico City Teaching Hospital
}

\author{
Jessica G. Azcorra-Lopez, Jesus Reyna-Figueroa, Eva M. Luna-Rivera, Berenice Cabañas-Espinosa, \\ Yamile N. Esquivel-Torruco, Arely A. Bejarano-Juvera, Aditi K. Bustamante-Flores, Naomi Aguilar- \\ Martinez, Daniel Lopez-Herrera, and Cesar A. Arce-Salinas
}

\section{ABSTRACT}

Introduction: During the COVID-19 pandemic, hospitals have been busy controlling their spread among healthcare workers, including resident doctors. We seek to characterize and know the transmission dynamics of the SARS-COV-2 infection in the personnel of our hospital.

Methodology: 204 resident physicians were followed up, in three parts: Part 1. Training, Part 2. follow-up through the WhatsApp application, Part 3. case-control study; looking for risk factors.

The statistical analysis was carried out using measures of central tendency and dispersion, comparison of proportions and rates. As well as the calculation of the odds ratio with a $95 \%$ confidence interval; $p<0.05$ was considered significant.

Results: 66 resident physicians were included, $31.8 \%$ had a positive result. The mean age was 28.9. The most frequent clinical manifestation was myalgia $(66.6 \%)$, followed by headache $(47 \%)$. When comparing with symptomatic resident physicians, with a negative test, there was a significant difference in arthralgia $(p=0.01)$ and dyspnea $(0.05)$.

Noncompliance with training $(\mathrm{OR} 7.0 ; 95 \%$ CI $2.2-26$ and $p=0.000)$ was significant as a risk factor for infection.

Conclusions: Resident physicians must be mostly supervised during infection prevention and containment training.

Keywords: COVID-19, resident physicians, training.

\section{INTRODUCTION}

Since COVID-19 was declared a pandemic, hospitals have tried to control its spread among healthcare workers, considered one of the groups with the highest numbers of contagion in the world [1]. In Mexico, until August 2020, considered.
Published Online: June 25, 2021

ISSN: $2736-5476$

DOI: 10.24018 /ejclinicmed.2021.2.3.36

Jessica G. Azcorra-Lopez*

Pediatrics Service, South Central

Hospital, Mexican Oils, Mexico City, Mexico.

(e-mail: jessica.al10@hotmail.com)

Jesus Reyna-Figueroa

Department of Teaching and Research, South Central Hospital, Mexican Oils, Mexico City, Mexico.

Eva M. Luna-Rivera

Department of Teaching and Research, South Central Hospital, Mexican Oils, Mexico City, Mexico.

Berenice Cabañas-Espinosa

Pediatrics Service, South Central Hospital, Mexican Oils, Mexico City, Mexico.

Yamile N. Esquivel-Torruco

Pediatrics Service, South Central Hospital, Mexican Oils, Mexico City, Mexico.

Arely A. Bejarano-Juvera

Pediatrics Service, South Central Hospital, Mexican Oils, Mexico City, Mexico.

Aditi K. Bustamante-Flores

Pediatrics Service, South Central Hospital; Mexican Oils. Mexico City, Mexico.

Naomi Aguilar-Martinez

Pediatrics Service, South Central Hospital, Mexican Oils, Mexico City, Mexico.

Daniel Lopez-Herrera

Work Medicine Service, South Central Hospital, Mexican Oils, Mexico City, Mexico.

Cesar A. Arce-Salinas

Directorate of South Central Hospital of High Specialty, Mexican Oils, Mexico City, Mexico.

*Corresponding Author $(1.3 \%)$ deaths; regardless of the contagion site (nosocomial or community), among which the resident doctors are

In addition to the educational problem that implies the suspension of academic programs as a consequence of 
prioritizing the care of patients with COVID-19; the modification of the role they play in a hospital is added; ranging from home confinement to direct care of patients with the disease [2], [3]. That is why training in prevention and the proper use of personal protective equipment (PPE) is considered a priority [4].

At the Hospital Central Sur de Alta Especialidad (HCSAE), a teaching hospital belonging to the Health Services of Mexican Oils; in Mexico, resident physicians comprise $17.1 \%$ of the 1,200 healthcare workers of the institution, they attend 1 of the 20 specialization courses that are taught there. From April 2020, once the institution was reconverted to care for patients with COVID-19, they were confined to their homes; dividing the hospital work by shifts to reduce the number and in-hospital flow of personnel. Furthermore, resident physicians with any of the known risk factors for the disease were not included in the hospital work.

The studies that evaluate the infection in this particular group of doctors are practically null, which is why we seek to characterize and know the transmission dynamics of the infection by SARS-COV-2 (COVID-19) in the HCSAE resident doctors.

\section{METHODOLOGY}

To fulfill the objective, we followed up 204 resident physicians, who were classified according to the orientation of the specialization courses in "clinical" (anatomypathology, imaging, internal medicine, occupational medicine, pediatrics, cardiology, critical medicine, nephrology) and "surgical" (anesthesiology, general surgery, ophthalmology, otorhinolaryngology, orthopedics, plastic and reconstructive surgery, neurosurgery, obstetricsgynecology).

The study was conducted in three parts:

Part 1. During April and May, the training of resident doctors was carried out on a mandatory basis, through the Microsoft Teams Office application, $100 \%$ being officially trained. Nevertheless, for this study, we consider resident physicians as trained, to those who met the following 3 points: a) full attendance throughout the meeting ratified by the meeting attendance report generated in the application used. The advantage of this application is that it records the name, join time, and leave time of all meeting attendees. b) to send photographic evidence and c) to attend at least $80 \%$ of the activities, without forgetting the training in the use of personal protective equipment.

Part 2. From April to October, the 204 residents of the HCSAE were followed up, through the WhatsApp application, intending to detect probable cases of COVID-19. A representative of the 15 courses that are taught in the hospital (the high specialty courses [5], were included in the core specialties), asked daily to their colleagues' specialists about their health condition; and when appropriate, if they had come to the institutional Respiratory Illness Clinic, with respiratory symptoms. All this was reported by the same means of communication to the chat group between the head of teaching and representatives of the specialty. Positive cases were identified by the SARS-COV-2 virus PCR test. His clinical evolution was followed through this same application and corroborated in the electronic clinical record. An attempt was made to demonstrate the transmission dynamics of the infection by investigating the in-hospital contacts, the time of presentation between one case and another and investigating the contact history, trying to determine the origin.

Part 3. In October, through a study of cases (resident doctors with positive PCR) and controls (resident doctors with suspected COVID-19, with negative PCR); We analyzed the clinical, laboratory, and cabinet characteristics of the group of infected residents vs probable cases; as well as the possible risk factors found.

The statistical analysis was carried out using measures of central tendency and dispersion, comparison of proportions and rates. As well as the calculation of the odds ratio with a $95 \%$ confidence interval; $\mathrm{p}<0.05$ was considered significant.

The study has been authorized by the HCSAE Research Ethics Committee.

\section{RESULTS}

\section{A. General Characteristics}

A total of 491 subjects from HCSAE with a positive test for SARS-CoV-2; 21 (4.2\%), corresponded to resident doctors, only $2 / 491(0.4 \%)$ were hospitalized, one in internal medicine and the other in intensive care unit, which corresponds to $9.5 \%$ of the 21 infected resident doctors. No deaths were reported in this group.

TABLE I: GENERAL CHARACTERISTICS OF RESIDENT PHYSICIANS ACCORDING TO THE RESULT OF THE DETECTION TEST FOR SARS-COV-2

\begin{tabular}{|c|c|c|c|}
\hline Variable & Cases (21) & Controls (45) & $\mathrm{p}$ \\
\hline Average age (DE) & $29 \pm 2.4$ & $28.9 \pm 2.3$ & 0.9 \\
\hline \multicolumn{4}{|l|}{ Gender $(\%)$} \\
\hline Men & 12 & 39 & \multirow{2}{*}{0.3} \\
\hline Women & 9 & 16 & \\
\hline Average BMI (DE) & $25.2 \pm 3.4$ & $25.1 \pm 3.4$ & 0.8 \\
\hline Overweight/obesity & 6 & 19 & 0.4 \\
\hline Chronic diseases $(\%)$ & 12 & 35 & 0.1 \\
\hline \multicolumn{4}{|l|}{ Specialty $(\%)$} \\
\hline Clinical & 10 & 29 & \multirow{2}{*}{0.3} \\
\hline Surgical & 11 & 16 & \\
\hline \multicolumn{4}{|l|}{ Source of contagion (\%) } \\
\hline Colleague & 1 & 4 & 0.9 \\
\hline Patient & 3 & 2 & 0.3 \\
\hline Relative & 3 & 10 & 0.6 \\
\hline Unknown & 14 & 29 & 1 \\
\hline $\begin{array}{l}\text { Average attention post-onset- } \\
\text { symptoms (interval) }\end{array}$ & $2(0-3)$ & $2(0-7)$ & 1 \\
\hline Hospitalizations (\%) & 2 & 0 & ------ \\
\hline Average viral load (interval) & $\begin{array}{c}712,720 \text { (506- } \\
15 \text { million) }\end{array}$ & 0 & ----- \\
\hline $\begin{array}{c}\text { Vaccination vs influenza } \\
2019(\%)\end{array}$ & 15 & 31 & 0.8 \\
\hline $\begin{array}{l}\text { Vaccination vs } \\
\text { pneumococcus during } \\
\text { residency }(\%)\end{array}$ & 5 & 3 & 0.1 \\
\hline Completed training (\%) & $4(19 \%)$ & $29(64 \%)$ & 0.001 \\
\hline
\end{tabular}

Finally, a total of 96 SARS-COV-2 tests were performed to 66 resident physicians (1.4 tests per physician). 2/66 $(31.8 \%)$ had a positive result. The proportion of infected men was $60 \%$ vs $40 \%$ of the female sex ( $<<0.05)$. The mean age was 28.9 years with a standard deviation (SD) of 2.6. The rest of the general characteristics, taking into account the result of the PCR test, are reported in Table I, in which it is seen that there were no differences between the group with COVID-19 vs the group with a negative test. Only a higher percentage of 
$89 \%$ vs $36 \%$ of non-compliance was observed in the training course, on the group with corroborated COVID-19 and the group with negative PCR, respectively.

The epidemic curve in which the rate of patients with positive COVID-19 in the Hospital Central Sur de Alta Especialidad (HCSAE), are compared with the rate of residents' positive to the test is shown in Fig. 1. The curves coincide closely, the diagnosis of COVID-19 in both groups reached its peak in April (39.4 and 2.7\% respectively) and the lowest rate in August, with a rebound in September. The average rate of positivity in those six months, about resident physicians was $3.5 \%$ vs. $28.3 \%$ in the rest of the patients. (p $=0.00001$ ).

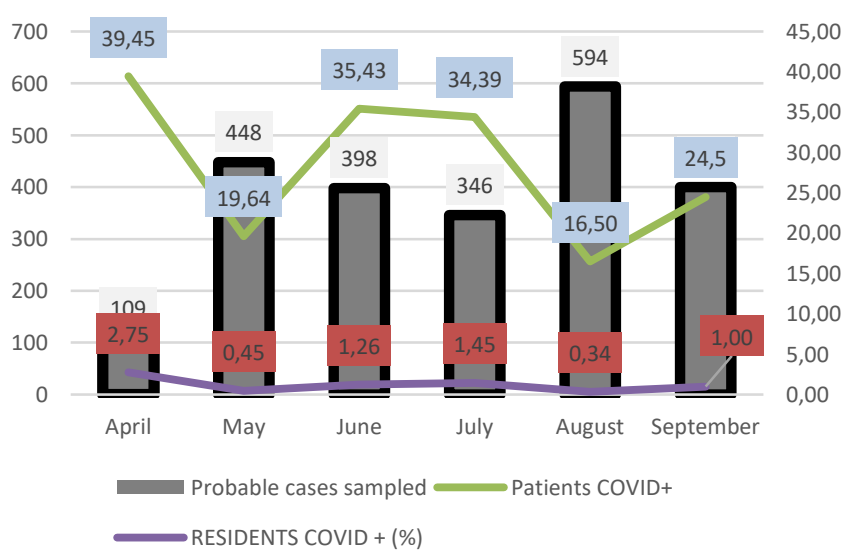

Fig. 1. Comparison of the positivity curve per month, between resident physicians and patients treated at the HCSAE.

\section{B. Clinical Picture}

The most frequent clinical manifestation found in SARSCOV-2-positive resident physicians was myalgias with $66.6 \%$, followed by headache in $47.6 \%$, odynophagia, and arthralgias (both with $42.8 \%$ ). When the comparative with symptomatic residents was done, but with the negative test, there was a significant difference in arthralgias $(\mathrm{p}=0.01)$ and the presence of dyspnea (0.05); in addition to radiographic changes, which only occurred in subjects with a positive test. With the rest of the symptoms, there was no difference in the percentage of presentation between both groups (see Table II).

TABLE II: COMPARISON OF THE CLINICAL MANIFESTATIONS PRESENTED IN THE GROUP OF RESIDENTS WITH A POSITIVE TEST VS RESIDENTS WITH A

\begin{tabular}{cccc}
\multicolumn{5}{c}{ NEGATIVE TEST } \\
\hline Variable & $\begin{array}{c}\text { Positive to } \\
\text { Covid (21) }\end{array}$ & $\begin{array}{c}\text { Negative to } \\
\text { Covid (45) }\end{array}$ & $\mathrm{p}$ \\
\hline Fever & $7(33.3)$ & $15(33.3)$ & 1.0 \\
Headache & $10(47.6)$ & $29(64.4)$ & 0.1 \\
Cough & $8(38)$ & $14(31)$ & 0.5 \\
Odynophagia & $9(42.8)$ & $17(37.7)$ & 0.6 \\
Myalgia & $14(66.6)$ & $24(53.3)$ & 0.3 \\
Arthralgia & $9(42.8)$ & $16(35.5)$ & 0.01 \\
Dyspnoea & $3(14.2)$ & $1(2.2)$ & 0.05 \\
Anosmia & $5(23.8)$ & $5(11.1)$ & 0.3 \\
Diarrhea & $4(19)$ & $12(26.6)$ & 0.7 \\
Self-medication & $7(33.3)$ & $9(20 \%)$ & 0.3 \\
Radiographic & $3(14.2)$ & 0 & --- \\
changes & & & - \\
\hline
\end{tabular}

\section{Risk Factors for COVID-19 in Resident Physicians}

In the univariate analysis, it was statistically significant for the lack of non-compliance in training with OR of 7.0 (2.226 ) and $p=0.000)$. Other factors such as obesity, the presence of chronic degenerative diseases (diabetes, hypertension, immunosuppression, asthma, or steroid intake included), or smoking were not significant as risk factors.

TABLE III: ANALYSIS OF RISK FACTORS FOR COVID-19 IN RESIDENT DOCTORS OF THE HOSPITAL CENTRAL SUR DE ALTA ESPECIALIDAD

\begin{tabular}{ccccc}
\hline Variable & $\begin{array}{c}\text { Cases } \\
(21)\end{array}$ & $\begin{array}{c}\text { Controls } \\
(45)\end{array}$ & OR (IC95\%) & $\mathrm{p}$ \\
\hline Obesity & 6 & 2 & $0.4(0.1-1.3)$ & 0.7 \\
Male gender & 10 & 30 & $0.4(0.1-1.3)$ & 0.2 \\
Hypertension & 2 & 3 & $1.4(0.2-9.5)$ & 1 \\
Immunosuppression & 1 & 0 & ---- & ---- \\
Use of steroids & 3 & 3 & $2.3(0.4-12)$ & 0.5 \\
$\quad$ Asthma & 3 & 10 & $0.5(0.1-2.7)$ & 0.5 \\
Smoking & 3 & 9 & $0.8(0.2-2.7)$ & 0.8 \\
Lack of training & $\mathbf{1 7}$ & $\mathbf{1 6}$ & $\mathbf{7 ( 2 . 2 - 2 6 )}$ & $\mathbf{0 . 0 0 0}$ \\
No vaccination against & 6 & 14 & $0.8(0.2-2.7)$ & 0.8 \\
influenza & & & & \\
No vaccination against & 16 & 42 & $4.3(0.9-20)$ & 0.1 \\
pneumococcus & & & &
\end{tabular}

\section{Dynamics of Infection}

The first two cases reported in resident physicians, occurred on April 24 and 25 in the course of pediatrics as part of an in-hospital outbreak as a result of contact with an attending who became infected with one of her relatives. Two days later, the case of an obstetrician-gynecologist resident was presented, which merited management in intensive care unit, not related to the previous ones.

About the 21 cases detected; in $3(14.2 \%)$ there was contact with an infected patient in the hospital, $3(14.2 \%)$ had contact with a colleague or healthcare worker infected outside the hospital, $6(28.5 \%)$ with a family member and $10(47.6 \%)$ the source of contagion was not identified (Fig. 2). During the course of the days, the phenomenon occurred that the coworkers of the positive resident doctors went to the Respiratory Illness Clinic referring symptoms such as headache or odynophagia; in no case was infection corroborated.

\begin{tabular}{|c|c|c|c|c|c|c|}
\hline Week & April & May & June & July & August & September \\
\hline $1-7$ & NO CASES & NO CASES & IM & NO CASES & IM & NO CASES \\
\hline 8-14 & NO CASES & NEURO & NO CASES & GYNEC & MAOG & NO CASES \\
\hline $15-21$ & NO CASES & ANESTH & $\left.\right|_{\text {GYNEC }} ^{\text {ANESTH }}$ & CRT & NO CASES & IMAG \\
\hline $22-28$ & $\begin{array}{l}\text { PED - PED } \\
\text { GYNEC }\end{array}$ & NO CASES & \begin{tabular}{|l|l|} 
ANESTH \\
GYNEC
\end{tabular} & PED & NO CASES & $\prod_{\text {IMAG }}$ NEPH \\
\hline
\end{tabular}

Fig. 2. Timeline in the case dynamics of medical residents with COVID19.

$\mathrm{PED}=$ Pediatrics,$\quad$ GYNEC $=$ Gynecology, $\quad$ NEURO=Neurosurgery, ANESTH=Anesthesiology, IM=Internal Medicine, CRIT=Critical Medicine, SURG=General Surgery, IMAG=Imaging, NEPH=Nephrology.

\section{DISCUSSION}

The present study represents the analysis of the behavior of an epidemic considered serious, in a group of healthcare workers with particular characteristics, on one hand, they are 
considered health professionals, once they have a certificate and university degree that endorses them as such, on the other hand, they are part of a group whose activities in preacademic conditions had the sole objective of learning specialty. When the COVID-19 pandemic began, there was concern about the risks to resident physicians and the adequacy of personal protective equipment [5], [6].

The main result of this study establishes that there is an association between the lack of training in protection and prevention against COVID-19 and the acquisition of the infection in resident doctors. The issue is complex, any regulatory authority would assume that a group accustomed to education and training would be pending and accomplish this part that is needed to contain the pandemic. Training, considered among the most important determinants of health, influences the capacity of medical care in terms of number of hospitals, beds, and healthcare workers that are available to deal with this virus, by avoiding infection; resulting in a greater number of assets being available to take care of it and in turn avoid being a source of infection (7). It is recognized that when health professionals have limited knowledge of an infectious disease, its prevention and its control; it contributes to its nosocomial spread [8], [9].

Although the training of health personnel, in general, has found in technology a way to solve the problem of not being able to have face-to-face training; There are barriers that organizations must overcome, such as ensuring that online attendees actually complete the training [10], [11].

On the other hand, infection acquired in the hospital can be considered an indicator of failure in the prevention and containment measures established by an organization [12]. In the group of resident doctors, where the role they play may be more moral than real [13], determine hospital-acquired cases should be considered a necessity, this will avoid panic among the healthcare workers themselves and can contribute to better emotional and mental stability [14]. For this, it may be useful to establish comparatively the dynamics of the epidemiological curve between the general population attended in the institution vs that observed in the infection of resident physicians [15], in our case this comparison establishes that the curves coincide closely, the diagnosis of COVID-19 in both groups peaked in April, and the lowest rate in August, with a rebound in September. This supports the fact that the largest number of resident physician cases were community-acquired.

Furthermore, COVID-19 is a viral disease that can affect all age groups, from infants to the elderly, resulting in a wide spectrum of various clinical manifestations; fever, dry cough, muscle weakness, and chest pain are the most common and typical symptoms of COVID-19 [16]. Our findings show that in this group, whose ages fall into that of patients with a low risk of complications [17], the main clinical manifestations were joint, muscular, and respiratory; These affectations can be explained by the immune activation and the response of cytokines in the alveolar and articular structures (the pattern of pro-inflammatory cytokines induced in COVID-19 has similarities with those directed in the treatment of rheumatoid arthritis). Although there are studies that establish that COVID-19 and other diseases mediated by zoonotic coronaviruses do not induce clinical arthritis [18], other authors report that there is enough evidence to say that muscle and joint symptoms are common and can even be considered an initial symptom of COVID-19 [19], the consideration to be evaluated is whether these manifestations occur more frequently in young subjects, with a lower rate of risk factors than in older people.

Despite our findings, some limitations should be considered. First, the sample size limits the establishment of whether any other risk factor has an impact on the acquisition of the disease; second, establishing the transmission dynamics of the disease in our population depends on the subject reminding or accurately referring the probable site of infection and contact with infected people.

This same situation actually depends on the population infection rate. It is known that the greater the circulation of the virus is, the more difficult it is to establish its origin.

Despite this, the results show us some weaknesses in the organizational containment and education measures; For example, it indicates that it is necessary to establish mechanisms with greater control so that we guarantee that the training meets its objective and that $100 \%$ of the population actually acquired the knowledge, giving importance to protection both in the hospital and in the community, where there appears to be a violation of the protocol. Also, greater emphasis should be placed on the epidemiological study of cases that occur in healthcare workers, in this case, resident physicians.

\section{CONCLUSIONS.}

Resident physicians are a group of healthcare workers who, from a prevention point of view; should be mostly supervised during training for the prevention and containment of SARSCOV-2 infection; because they fall into non-fulfillment in a significant percentage, and it can be a risk factor for acquiring the infection.

The transmission dynamics, at least in the hospital, establish that the highest proportion of infection in this specific group was community-acquired. The evolution of the disease was benign in all cases, recovery was $100 \%$, with only two cases requiring hospitalization, and only one of them required mechanical ventilatory support. Arthralgias and dyspnea were the symptoms most commonly referred to in the clinical picture.

\section{REFERENCES}

[1] Zheng C, Hafezi-Bakhtiari N, Cooper V, Davidson H, Habibi M, Riley $\mathrm{P}$, Breathnach A. Characteristics, and transmission dynamics of COVID-19 in healthcare workers at a London teaching hospital. J Hosp Infect. 2020;106(2):325-329.

[2] Ahmed H, Allaf M, Elghazaly H. COVID-19 and medical education Lancet Infect Dis. 2020;20(7):777-778.

[3] Cram P, Anderson ML, Shaughnessy EE. All Hands-on Deck: Learning to "Un-specialize" in the COVID-19 Pandemic. J Hosp Med. 2020;15(5):314-315.

[4] Cook TM. Personal protective equipment during the coronavirus disease (COVID) 2019 pandemic - a narrative review. Anaesthesia. 2020;75(7):920-927.

[5] Characteristics and transmission dynamics of COVID-19Journal of Hospital Infection 106 (2020) 325e329.

[6] O'Byrne L, Gavin B, McNicholas F. Medical students and COVID-19: the need for pandemic preparedness. J Med Ethics. 2020;46(9):623626. 
[7] Noorbhai H. A mathematical model to guide the re-opening of economies during the COVID-19 pandemic. Ann Med Surg (Lond). 2020;57:5-6

[8] Ma H, Zhu J, Liu J, Zhang X, Liu Y, Yang Q. Hospital biosecurity capacitation: Analysis and recommendations for the prevention and control of COVID-19. J Biosaf Biosecur. 2020 ;2(1):5-9.

[9] Shrestha S. Teaching and Training Medical Students at the Midst of COVID-19 Pandemic: Unanswered Questions and the Way Forward. J Nepal Health Res Counc. 2020;18(2):320-323.

[10] Grange ES, Neil EJ, Stoffel M, Singh AP, Tseng E, Resco-Summers K, Fellner BJ, Lynch JB, Mathias PC, Mauritz-Miller K, Sutton PR, Leu MG. Responding to COVID-19: The UW Medicine Information Technology Services Experience. Appl Clin Inform. 2020;11(2):265 275.

[11] Santoro E. Information technology e digital health a supporto della salute ai tempi della CoViD-19 [Information technology and digital health to support health in the time of CoViD-19.]. Recenti Prog Med. 2020;111(7):393-397).

[12] Alpalhão V, Alpalhão M. Impact of COVID-19 on Physical Therapist Practice in Portugal. Phys Ther. 2020 Jul 19;100(7):1052-1053.

[13] Reyna-Figueroa J, Bejarano-Juvera AA, Arce-Salinas CA, MartínezArredondo H, Lehmann-Mendoza R. Opportunities in the teaching of medical specialties, regarding influenza and COVID-19. Gac Med Mex. 2020;156(4):321-327.
[14] Sharif S, Amin F, Hafiz M, Benzel E, Peev N, Dahlan RH, Enchev Y, Pereira P, Vaishya S; World Spinal Column Society Executive Board. COVID 19-Depression and Neurosurgeons. World Neurosurg. 2020;140:e401-e410.

[15] Menon A, Klein EJ, Kollars K, Kleinhenz ALW. Medical Students Are Not Essential Workers: Examining Institutional Responsibility During the COVID-19 Pandemic. Acad Med. 2020;95(8):1149-1151.

[16] Baj J, Karakuła-Juchnowicz H, Teresiński G, Buszewicz G, Ciesielka M, Sitarz E, Forma A, Karakuła K, Flieger W, Portincasa P, Maciejewski R. COVID-19: Specific and Non-Specific Clinical Manifestations and Symptoms: The Current State of Knowledge. J Clin Med. 2020;9(6):1753.

[17] Palaiodimos L, Kokkinidis DG, Li W, Karamanis D, Ognibene J, Arora S, Southern WN, Mantzoros CS. Severe obesity, increasing age, and male sex are independently associated with worse in-hospital outcomes, and higher in-hospital mortality, in a cohort of patients with COVID-19 in the Bronx, New York. Metabolism. 2020;108:154262.

[18] Schett G, Manger B, Simon D, Caporali R. COVID-19 revisiting inflammatory pathways of arthritis. Nat Rev Rheumatol. 2020;16(8):465-470.

[19] Joob B, Wiwanitkit V. Arthralgia as an initial presentation of COVID19: observation. Rheumatol Int. 2020;40(5):823. 